\title{
IN VITRO SCREENING OF SOYBEAN GENOTYPES UNDER SALINITY STRESS
}

\author{
M.K. Hasan ${ }^{1 *}$, K.M. Nasiruddin ${ }^{2}$, M. Al-Amin ${ }^{3}$ and A.K.M.S. Hossain ${ }^{4}$ \\ ${ }^{1}$ Biotechnology Division, Bangladesh Agricultural Research Institute, Gazipur, Bangladesh. \\ ${ }^{2}$ Department of Biotechnology, Bangladesh Agricultural University, Mymensingh, Bangladesh. \\ ${ }^{3}$ Supreme Seed Company, Uttara, Dhaka, Bangladesh. \\ ${ }^{4}$ Farm Management Unit, Bangladesh Jute Research Institute, Dhaka, Bangladesh.
}

*Corresponding author's email: kamrulnk@yahoo.com

\begin{abstract}
Salinity is one of the most limiting factors for successful crop production in in arid and semi-arid regions of the world. Thirty eight soybean genotypes were screened at $8 \mathrm{mMNaCl}$ under in vitro condition. Salinity reduced Shoot dry weight, Root dry weight and Plant height. Salt susceptibility index was fully and positive correlated with percent reduction of total dry weight. Principal component analysis showed that the first two components were extracted that comprises of about $98.6 \%$ of the total variation in the genotypes. Based on the K-means clustering, 8, 6, 12 and 12 genotypes were categorized under cluster II, IV, III and I and considered as tolerant, moderately tolerant, moderately susceptible and susceptible which represents the 21, 16, 31.5 and 31.5\%, respectively. Genotypes Shohag, AGS 313, PK 416, AGS 66, MACS 57, AGS 195, GC 308 , AGS 129 were found relatively tolerant to salinity.
\end{abstract}

Keywords: Soybean; salinity; in vitro screening.

\section{Introduction}

Soybean (Glycine $\max$ L. Merrill) has a variety of uses including for vegetable oil, human food, livestock feed and many industrial products. Soil salinity is a global environmental challenge, affecting crop production on over 800 million hectares, or a quarter to a third of all agricultural land on earth (Rengasamy, 2010). Soybean has been considered as a salt sensitive to moderately salt-tolerant crop (Luo et al., 2005). Screening is most important for the identification of salt tolerant genetic resources. Salinity tolerance test in various crops have been carried out by different scientists in different ways. To evaluate salinity tolerance, a number of models for the response of plants to salinity have been defined. Although salinity tolerance tests are being carried out directly in the saline soils, but it is difficult to maintain the exact level of salinity. Because with the advancement of time and plant growth there would be constant change in the macroclimate conditions i.e. loss of moisture from soil which increases the salinity level at the root level of the crop being grown. Field screening procedures in saline soils are confronted by high spatial and temporal variability problems. In vitro culture is an ideal system for evaluating saline tolerant plants as it can be carried out under controlled conditions with limited space and time (Munns et al., 2006) and found suitable to screen some other crops (Vijayan et al., 2003; Erturk et al., 2007; Govindaraj et al., 2010; Dasgupta et al., 2008; Mungala et al., 2008; Bhute et al., 2012). The present study was carried out to screen the soybean genotypes for salt tolerance at early vegetative stage under in vitro condition.

\section{Materials and Methods}

Thirty eight genotypes of soybean were studied in this experiment collected from Oilseed Research Centre, Bangladesh Agricultural Research Institute, Gazipur, Bangladesh. Mature and uniform seeds of soybean were washed in running tap water for 3-5 minutes to reduce the level of surface organism. The floating seeds were discarded. Then seeds were cleaned with Tween 20 (1-2 drops) and washed by sterile water (2-3 times). Cleaned seeds were dipped in $70 \%$ alcohol for 2-3 minutes with vigorous shaking followed by washing by sterile distilled water (2-3 times). Surface disinfection was done by $4 \%$ $\mathrm{NaOCl}$ solution for 5 minutes. The surface sterilized seeds were then placed in half strength of MS Media (Murashige and Skoog, 1962) with $80 \mathrm{mM} \mathrm{NaCl}$ and without $\mathrm{NaCl}$ (control) and kept in dark condition with control temperature $\left(25 \pm 1^{\circ} \mathrm{C}\right)$ for about $2-3$ days. Then the test tubes were transferred to light intensity of 2000-3000 lux from fluorescent tube. The photoperiod was maintained at 16 hours 
light and 8 hours dark (16L/8D) and the relative humidity was $60-70 \%$. After four weeks, all these seedlings of each vial were removed for further observation and data recording. Plant height was measured from tip of the root to tip of growing region after that plants were separated into roots and shoots. For dry weight, the samples were kept at $70^{\circ} \mathrm{C}$ for 48 hours. Plant dry weight under salt stress and non-stress treatment were determined. Geometric mean (GM) was determined as $\mathrm{GM}=(\mathrm{NS} \times \mathrm{SS})^{1 / 2}$ where, $\mathrm{NS}=$ non stress and $\mathrm{SS}=$ salt stress. Percentage of reduction (PR) and relative total dry weight (RTDW) due to salinity stress in relation to the non-stress environment were determined for the two traits. Salinity susceptible index (SSI) for total dry weight of each genotype was calculated following Fisher and Mauer (1978) as follows:

$\mathrm{SSI}=(1-\mathrm{Yss} / \mathrm{Yns}) / \mathrm{SII}$

Where, Yss =Total dry weight of a genotype under salt stress

Yns = Total dry weight of a genotype under non stress

SII (salinity intensity index $)=(1-\mathrm{Xss} / \mathrm{Xns})$

Xss = Mean of the total dry weight of all accession under salinity stress

$\mathrm{Xns}=$ Mean of the total dry weight of all accession under non stress

Mean data for each character was analyzed by multivariate analysis using computer software GENSTAT-5.
Correlation coefficient was also done through the software SPSS version 12.0.

\section{Results and Discussion}

\section{Variability in Quantitative Characters}

Due to salinity, the reduction of shoot dry weight, root dry weight, total dry weight and plant height were $37,41,38$ and $47 \%$, respectively (Table 1 ). The root/shoot ratio of total dry weight under control and salt treatment were very close. Variations were found among the different characters under control, but it was narrow under the salt stress condition revealed that soybean genotypes were much influenced by salt stress. Mannan et al. (2010) found the similar results. Due to imposition of salinity, the highest reduction was observed in total dry weight. The detrimental effect of salt is generally observed at the whole plant level. Dry matter accumulation of different plant parts are severely affected by high level of salinity that ultimately reduce crop yield (Change et al., 1994). Effect of salinity was more in relative root dry weight than that of relative shoot dry weight. Similar phenomenon was found by Essa (2002).Wide range variation in different characteristics (shoot dry weight, root dry weight, total dry weight, root/shoot ratio, plant height) in relative performance basis, geometric mean, per cent reduction and salinity susceptible index of total dry weight of soybean genotypes were in salinity stress condition (Table 2).

Table 1: Range and mean for quantitative characters of 38 soybean genotypes subjected to salt stress

\begin{tabular}{lclll}
\hline \multirow{2}{*}{ Plant characters } & \multicolumn{2}{c}{ Control } & \multicolumn{2}{c}{ Salt treated $^{\mathbf{1}}$} \\
\cline { 2 - 5 } & Range & Mean & Range & Mean $^{\mathbf{2}}$ \\
\hline Shoot dry weight $\left(\mathrm{mg} \mathrm{plant}^{-1}\right)$ & $53-145$ & $89.45 \pm 25.92$ & $26-117$ & $56.14 \pm 25.23$ \\
Root dry weight $\left(\mathrm{mg} \mathrm{plant}^{-1}\right)$ & $12-43$ & $23.57 \pm 7.89$ & $6-35$ & $13.93 \pm 7.39$ \\
Total dry weight $\left(\mathrm{mg} \mathrm{plant}^{-1}\right)$ & $70-169$ & $113.01 \pm 28.77$ & $32-131$ & $70.11 \pm 29.88$ \\
Root/shoot ratio & $0.10-0.46$ & $0.28 \pm 0.09$ & $0.10-0.43$ & $0.26 \pm 0.09$ \\
Plant height $(\mathrm{cm})$ & $9.1-18.93$ & $13.69 \pm 2.72$ & $4.88-12.56$ & $7.23 \pm 2.13$ \\
\hline
\end{tabular}

1 plants were allowed to grow at MS medium with $80 \mathrm{mM} \mathrm{NaCl}$ for 4 weeks.

${ }^{2}$ mean \pm standard deviation

Table 2: Range and mean for relative plant characters, geometric mean, per cent reduction and salinity susceptibility index of soybean genotypes subjected to salt stress

\begin{tabular}{lll}
\hline Characters & Range (\%) & Mean (\%) \\
\hline Relative shoot dry weight & $39.24-84.75$ & $61.09 \pm 13.90$ \\
Relative root dry weight & $31.58-91.67$ & $57.91 \pm 15.87$ \\
Relative total dry weight & $39.02-82.93$ & $60.36 \pm 13.83$ \\
Geometric mean of total dry weight & $51.22-147.65$ & $88.45 \pm 29.47$ \\
Per cent reduction of total dry weight & $17.07-60.98$ & $39.64 \pm 13.83$ \\
Salinity susceptibility index & $0.45-1.61$ & $1.04 \pm 0.36$ \\
Relative root /shoot ratio & $61.97-141.63$ & $95.17 \pm 17.80$ \\
Relative plant height & $34.41-66.35$ & $52.26 \pm 7.30$
\end{tabular}


M.K. Hasan et al. (2016) Int J Appl Sci Biotechnol, Vol 4(2): 207-212

Table 3: Correlation coefficients among the different characters for 38 soybean genotypes

\begin{tabular}{|c|c|c|c|c|c|c|c|c|c|c|c|c|c|c|c|c|c|c|}
\hline & \multicolumn{4}{|c|}{ Shoot dry weight } & \multicolumn{3}{|c|}{ Root dry weight } & \multicolumn{6}{|c|}{ Total dry weight } & \multicolumn{3}{|c|}{ Root/Shoot Ratio } & \multicolumn{2}{|c|}{ Plant height } \\
\hline & & NS & SS & Rel & NS & SS & Rel & NS & SS & Rel & GM & SSI & $\overline{\text { PR }}$ & $\mathbf{N S}$ & SS & Rel & NS & SS \\
\hline \multirow{3}{*}{ Shoot dry weight } & NS & - & & & & & & & & & & & & & & & & \\
\hline & SS & $0.893^{* *}$ & - & & & & & & & & & & & & & & & \\
\hline & Rel & $0.424 * *$ & $0.771 * *$ & - & & & & & & & & & & & & & & \\
\hline \multirow{3}{*}{ Root dry weight } & NS & 0.228 & $0.327 *$ & $0.325^{*}$ & - & & & & & & & & & & & & & \\
\hline & SS & $0.355^{*}$ & $0.543^{* *}$ & $0.631^{* *}$ & $0.847^{* * *}$ & - & & & & & & & & & & & & \\
\hline & Rel & $0.396^{*}$ & $0.612 * *$ & $0.762^{* *}$ & 0.232 & $0.688^{* *}$ & - & & & & & & & & & & & \\
\hline \multirow{6}{*}{ Total dry weight } & NS & $0.964 * *$ & $0.895^{* *}$ & $0.472 * *$ & $0.480^{* *}$ & $0.553^{* *}$ & 0.420 ** & - & & & & & & & & & & \\
\hline & SS & $0.841^{* *}$ & $0.978^{* *}$ & $0.806^{* *}$ & $0.486^{* * *}$ & $0.706^{* * *}$ & $0.687 * *$ & $0.891^{* *}$ & - & & & & & & & & & \\
\hline & Rel & $0.450^{* * *}$ & $0.778^{* *}$ & $0.99 * *$ & 0.320 & $0.671^{* * *}$ & $0.842 * *$ & 0.493 ** & $0.823^{* *}$ & - & & & & & & & & \\
\hline & GM & $0.911 * *$ & $0.972^{* *}$ & $0.702^{* *}$ & $0.493 * *$ & $0.665^{* * *}$ & $0.606^{* * *}$ & $0.956^{* * *}$ & $0.985^{* *}$ & $0.721 * *$ & - & & & & & & & \\
\hline & SSI & $-0.444 * *$ & $-0.776 * *$ & $-0.990^{* * *}$ & -0.317 & $-0.668 * *$ & $-0.840 * *$ & $-0.487 * *$ & -0.820 ** & $-1.000^{* * *}$ & $-0.717 * *$ & - & & & & & & \\
\hline & PR & $-0.444 * *$ & $-0.775 * *$ & $-0.909 * *$ & -0.316 & $-0.668 * *$ & $-0.841 * *$ & $-0.487 * *$ & $-0.820^{* * *}$ & $-1.000^{* * *}$ & $-0.716^{* * *}$ & $1.000^{* *}$ & - & & & & & \\
\hline \multirow{3}{*}{ Root/ Shoot Ratio } & NS & $-0.510^{* * *}$ & $-0.350^{*}$ & -0.240 & $0.678^{* * *}$ & $0.420^{\text {** }}$ & -0.114 & -0.274 & -0.190 & -0.057 & -0.231 & 0.055 & 0.056 & - & & & & \\
\hline & SS & $-0.443 * *$ & -0.317 & -0.015 & $0.642^{* * *}$ & $0.567^{* *}$ & 0.184 & -0.223 & -0.125 & 0.019 & -0.168 & -0.020 & -0.020 & $0.879^{* *}$ & - & & & \\
\hline & Rel & 0.109 & -0.011 & -0.100 & -0.098 & 0.223 & $0.552^{* * *}$ & 0.071 & 0.046 & 0.035 & 0.059 & -0.033 & -0.035 & -0.209 & 0.260 & - & & \\
\hline \multirow{3}{*}{ Plant height } & NS & 0.264 & $0.546^{* *}$ & $0.708^{* *}$ & $0.405^{*}$ & $0.579^{* *}$ & $0.480^{* *}$ & $0.349 *$ & $0.603^{* *}$ & $0.688^{* *}$ & $0.519^{* *}$ & $-0.690^{* *}$ & $-0.689 * *$ & 0.135 & 0.101 & -0.167 & - & \\
\hline & SS & $0.346^{*}$ & $0.659^{* *}$ & $0.839^{* *}$ & $0.481^{* * *}$ & $0.700^{* *}$ & $0.606^{* * *}$ & $0.444^{* *}$ & $0.729^{* *}$ & $0.823^{* *}$ & $0.636^{* *}$ & $-0.824 * *$ & $-0.824 * *$ & 0.133 & 0.117 & -0.151 & $0.881^{* *}$ & - \\
\hline & Rel & $0.327^{*}$ & $0.557^{* *}$ & $0.687^{* *}$ & $0.360^{*}$ & $0.545^{* *}$ & $0.516^{* *}$ & $0.394 *$ & $0.605^{* *}$ & $0.679 * *$ & $0.539 * *$ & $-0.679^{* * *}$ & $-0.680^{* *}$ & -0.045 & 0.047 & -0.096 & $0.360^{*}$ & $0.754 * *$ \\
\hline
\end{tabular}

NS= Non salt, $S S=$ Salt stress, GM= Geometric mean, PR= Percent reduction, SSI= Salinity susceptibility index, Rel =Relative 


\section{Correlation Coefficient}

The degree of association among the different characters was determined by using Pearson's coefficient (Table 3). Salt susceptibility index is fully and positive correlated $(\mathrm{r}=$ $1.00 * *$ ) with percent reduction of total dry weight. Fully but negative correlation was found between relative total dry weight and salinity susceptibility index $\left(r=-1.00^{* *}\right)$ and between relative total dry weight and percent reduction of total dry weight $\left(\mathrm{r}=-1.00^{* *}\right)$. Lower and insignificant correlation was observed between total dry weight and root/shoot ratio. Shoot dry weight was more correlated $(r=$ $0.99 * *)$ with total dry weight than root dry weight $(\mathrm{r}=$ $0.842 * *)$. It also found a positive and significant correlation between relative plant height and total dry weight $(\mathrm{r}=$ $0.679 * *)$. The geometric mean for total dry weight in

\section{Principal Component Analysis}

The Eigen values associated with each principal component (PC) estimates the contribution of each PC to the total variance (Table 4). The first two components were extracted that comprises of about $98.6 \%$ of the total variation in the genotypes. The first component accounted for $95.38 \%$ of the total variation and $3.26 \%$ was in the second components. PC1 was heavily weighted by relative total dry weight, percent reduction of total dry weight and salinity susceptibility index. Second component was contributed due to relative root dry weight and relative root/shoot ratio. Negative contribution of percent reduction of total dry weight and salinity susceptibility index was found in the salinized and control conditions were positively correlated with shoot and root dry weight. Salinity susceptibility index was positively and fully correlated with percent reduction of total dry weight. Similar associations were found by Bayuelo-Jimenez et al. (2002). Negative correlation of total dry matter with percent reduction and salinity susceptibility index indicating that lower value of percent reduction and salinity susceptibility index are more acceptable for tolerance. As lower and insignificant correlation between total dry weight and root/shoot ratio indicating that root/shoot ratio has no significant effect on the total dry weight. Due to higher value of correlation, relative shoot dry weight was more correlated with salinity susceptible index (SSI) than root dry weight i.e. shoot dry weight has great influence on the SSI.

first components while relative shoot dry weight, relative total dry weight, geometric mean of total dry weight and relative plant height in the second components.

Principal Component (PC) analysis showed that the first two components were extracted that comprises of about $98.6 \%$. This value was near to the finding of Mannan et al. (2010) who found that $99.5 \%$ of the total variation in the genotypes by PC values. Gawande et al. (2002) also studied fifty genotypes of soybean including both indigenous and exotic lines for genetic diversity and found $93.53 \%$ of the total variation for ten quantitative characters accounted by the first three canonical vectors.

Table 4: Extracted Eigen values and latent vectors associated with the first two principal components

\begin{tabular}{|l|l|l|}
\hline \multirow{2}{*}{} & \multicolumn{2}{|c|}{ Principal components } \\
\cline { 2 - 3 } & 1 2nd \\
\hline Extracted Eigen values & 25.017 & 0.854 \\
\hline Percentage variation & 95.38 & 3.26 \\
\hline Latent vectors & & \\
\hline Relative shoot dry weight & & -0.1564 \\
\hline Relative root dry weight & 0.4032 & 0.4232 \\
\hline Relative total dry weight & 0.3597 & -0.0401 \\
\hline Geometric mean of total dry weight & 0.4111 & -0.0209 \\
\hline Percent reduction of total dry weight & 0.3247 & 0.0404 \\
\hline Salinity susceptibility index & -0.4109 & 0.0420 \\
\hline Relative root/shoot ratio & & 0.8627 \\
\hline Relative plant height & -0.4108 & -0.2161 \\
\hline
\end{tabular}




\section{Cluster Analysis}

On the basis of variation, genotypes were grouped into four cluster using K-means non-hierarchical cluster analysis (Table 4). Relative shoot dry weight, relative root dry weight, relative total dry weight, geometric mean of total dry weight and relative plant height were highest in the cluster II, lowest in the cluster I and moderate in cluster III and IV. Contrarily, percent reduction of total dry weight and salinity susceptibility index were lowest in II and highest in the cluster I and moderate in the III and IV. Relative root/shoot ratio were same at the cluster group II and III while relative root/shoot ratio was lowest in cluster I and highest in cluster IV. Relative plant height was also lowest at cluster I. Overall, cluster IV showed slightly better than III. Hence, cluster II may be considered as tolerant and cluster I may be considered as susceptible. Comparing cluster IV with III, a little relative shoot dry matter and relative plant height were higher in the cluster III, but other characters like relative root dry weight, relative total dry weight and geometric mean of total dry weight were higher in cluster IV. Two important characters- percent reduction of total dry weight and salt susceptible index were lower in the cluster IV indicates its superiority than cluster III. Moreover, distance matrix $\left(\mathrm{D}^{2}\right)$ showed that cluster II was close to cluster IV (4.03) and far from III (9.24). Therefore, the genotypes grouped in cluster IV and III may be considered as moderately tolerant and moderately susceptible, respectively. Distance matrix $\left(\mathrm{D}^{2}\right)$ showed that the four clusters were statistically different from each other. Similar $\mathrm{D}^{2}$ analysis was used to identify the distance between clusters by other authors (Mannan et al., 2010; Islam, 2011).

\section{Grouping of the Genotypes in Different Groups}

Genotypes Shohag, AGS 313, PK 416, AGS 66, MACS 57, AGS 195, GC 308 and AGS 129 were found as tolerant AGS 335, MTD 16, MTD 453, BARI Soybean 5, Asset 93, MTD 455 as moderately tolerant, K 16, Galarsin, Bangladesh soybean 4, Joyawiya, GC 108-1, GC 83001, Keratar, MTD 451, AGS 154, BARI Soybean 6, Davis, TGX-573-2 as moderately susceptible and No. 205, GMOT 17, UPSM, G 2261, AGS 314, IACHONO, Sholv, PI417475, Kadarsin, Asset 95, YESSOY 4 and AGS 302 were found as susceptible. The findings are corroborated with other researchers. Datta et al. (2006) found the Asset 93, Shohag and ASG 302 as salt tolerant in a pot culture experiment while Aziz et al. (2009) found BARI Soybean 5, Shoahg and MACS 57 along with other some genotypes performed better upto 8dSm${ }^{-1}$. Islam (2005) conducted an experiment on laboratory (in vitro) screening of 14 soybean genotypes for salt tolerance at seedling stage and found that G 2120, Asset 93 and TG 88 were more tolerant to salinity than others. Mannan et al. (2009) conferred the AGS 313 as relatively salt tolerant in respect of dry matter accumulation compared to other genotypes. Considering the salinity susceptibility index, Khan et al. (2012) also found Shohag, AGS 313 and PK 416 as tolerant.

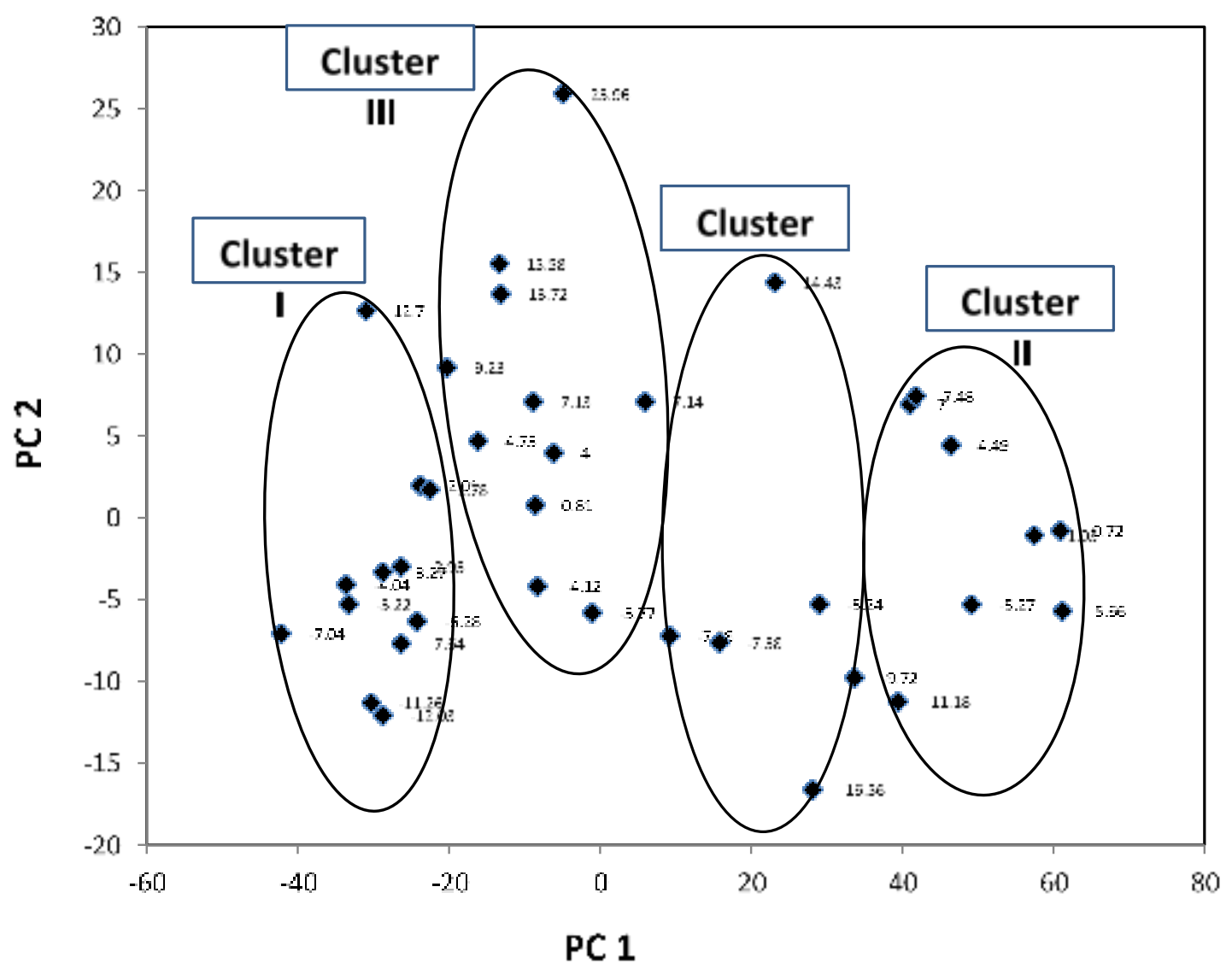

Fig. 1: Grouping of soybean genotypes subjected to salinity by PCA 


\section{Conclusion}

In the present study, the in vitro screening technique was used to find out the salt tolerant soybean genotypes in different groups. The findings are corroborated by others indicating that the technique followed in this study may be utilized as an effective method of screening for salt tolerance.

\section{References}

Aziz MA, Begum F and Saha RR (2009) Technical and Financial Progress Report of core Research Programs/Projects implemented during the period from 2006-07 to 2008-09 under 'Research grant' of BARC on Studies on salt tolerance mechanism and development of salt tolerant varieties/technology of different crops/cultivars for coastal area of Bangladesh. Agronomy Division, BARI, Gazipur. pp. 33-44.

Bayuelo-Jimenez JS, Debouck DG and Lynch JP (2002) Salinity tolerance in Phaseolus species during early vegetative growth. Crop Sci. 42: 2184-2192. DOI: 10.2135/cropsci2002.2184

Bhute N, Vamadevaih HM, Katageri IS, Uppainal NF and Mirajkar KK (2012) In vitro screening for salinity stress at seedling stage of cotton. Karnataka J. Agril. Sci.25 (1): 39 4.

Change RZ, Chen YW, Shao GH and Wan CW (1994) Effect of salt stress on agronomic characters and chemical quality of seeds in soybean. Soybean Sci. 13: 101-105.

Dasgupta M, Sahoo MR, Kole PC and Mukherjee A (2008) Evaluation of orange-fleshed sweet potato (Ipomoea batatas L.) genotypes for salt tolerance through shoot apex culture under in vitro $\mathrm{NaCl}$ mediated salinity stress conditions. Plant Cell Tissue and Organ Culture. 94: 161170. DOI 10.1007/s 11240-008-9400-2

Datta AK, Hossain MA, Robin ABMAHK, Raffi SA and Hossain MA (2006) Screening of soybean genotypes for salinity tolerance at seedling stage. Bangladesh J Crop Sci. 17(1): 155-161.

Erturk U, Sivritepe N, Yerlikaya C, Bor M, Ozdemir F and Turkan I (2007) Responses of the cherry rootstock to salinity in vitro. Biologia Plant. 51(3): 597-600. DOI: 10.1007/s10535-007-0132-7

Essa TA (2002) Effect of Salinity Stress on Growth and Nutrient Composition of Three Soybean (Glycine max L. Merrill) Cultivars. J. Agron. Crop. Sci.188: 86-93. DOI: 10.1046/j.1439-037X.2002.00537.x

Fisher RA and Maurer R (1978) Drought resistance in spring wheat cultivars: Grain yield response. Australian J. Agril Res. 29: 897-912. DOI: 10.1071/AR9780897

Gawande PP, Patil S, Mayakumari and Bailkiwad VJ (2002) Selection of superior germplasm lines of soybean Glycine max (L) Merrill for breeding. J Soils Crops. 12(2): 236240.

Govindaraj M, Shanmugasundaram P, Sumathi P and Muthiah AR (2010) Simple, rapid and cost effective screening method for drought resistant breeding in pearl millet. Electronic $J$. Plant Breeding. 1(4): 590- 599.

Islam MM (2005) Laboratory screening of Soybean (Glycine max) genotypes for salt tolerance and in vitro regeneration of the selected genotypes, MS Thesis, Department of Genetics and Plant Breeding, Bangladesh Agricultural University, Mymensingh.

Islam MT (2011) Morpho-Molecular characterization and in vitro regeneration of hyacinth bean (Lablab purpureus), $\mathrm{PhD}$ Thesis. Dept. of Biotechnology, Bangladesh Agricultural University, Mymensingh, Bangladesh.

Khan MSA, Karim MA, Haque MM, Karim AJMS and Mian MAK (2012) Screening of soybean genotypes for salt tolerance in hydroponics. Paper presented at the conference on 'Advances in agronomic research under changing environment in Bangladesh' held on October 6, 2012 at Bangladesh Agricultural Research Institute, Gazipur, Bangladesh.

Luo Q Yu B and LiuY (2005) Differential sensitivity to chloride and sodium ions in seedling of Glycine max and G. soja under $\mathrm{NaCl}$ stress. J Plant Physiol. 162: 1003-1012. DOI: 10.1016/j.jplph.2004.11.008

Mannan MA, Karim MA, Khaliq QA and Ahmed JU (2009) Salinity effect on dry matter accumulation in different vegetative parts of soybean. J. Bangladesh Society Agril. Sci and. Tech. 6(1.\& 2):35-38.

Mannan MA, Karim MA, Khaliq QA, Haque MM, Mian MAK and Ahmed JU (2010) Assessment of genetic divergence in salt tolerance of soybean (Glycine max L.) genotypes. $J$ Crop Sci Biotech. 13 (1):130-134. DOI: 10.1007/s12892009-0091-y

Mungala AJ, Radhakrishnan T and Dobaria JR (2008) In vitro Screening of 123 Indian peanut cultivars for sodium chloride induced salinity tolerance. World J. Agril. Sci. 4(5): $574-582$

Munns R, James RA and Lauchli A (2006) Approaches to increasing the salt tolerance of wheat and other cereals. Exp. J. Bot. 57: 1025-1043. DOI: 10.1093/jxb/erj100

Murashige T and Skoog F (1962) A revised medium for rapid growth and bioassay with tobacco tissue cultures. Physiol Plan. 15: 473-497. DOI: 10.1111/j.13993054.1962.tb08052.x

Rengasamy P (2010) Soil processes affecting crop production in salt-affected soils. Func. Plant Biol. 37: 613-620.

Vijayan K, Chakraborti SP and Ghosh PD (2003) In vitro screening of mulberry (Morus spp.) for salinity tolerance. Plant Cell Report. 22: 350-357. DOI: 10.1007/s00299003-0695-5 